TRANSACTIONS OF THE

AMERICAN MATHEMATICAL SOCIETY

Volume 350, Number 8, August 1998, Pages 3169-3191

S 0002-9947(98)02303-4

\title{
SIMILARITY TO A CONTRACTION, FOR POWER-BOUNDED OPERATORS WITH FINITE PERIPHERAL SPECTRUM
}

\author{
RALPH DELAUBENFELS
}

\begin{abstract}
Suppose $T$ is a power-bounded linear opertor on a Hilbert space with finite peripheral spectrum (spectrum on the unit circle). Several sufficient conditions are given for $T$ to be similar to a contraction. A natural growth condition on the resolvent in half-planes tangent to the unit circle at the peripheral spectrum is shown to be equivalent to $T$ having an $H^{\infty}(\mathcal{P}) \cap C(\overline{\mathcal{P}})$ functional calculus, for some open polygon $\mathcal{P}$ contained in the unit disc, which, in turn, is equivalent to $T$ being similar to a contraction with numerical range contained in a closed polygon in the closed unit disc. Having certain orbits of $T$ be square summable also implies that $T$ is similar to a contraction.
\end{abstract}

\section{INTRODUCTION}

Suppose $T$ is a bounded linear operator on a Hilbert space $H$. Von Neumann's inequality (see, for example, [Be, Proposition X.1.7]) states that, if $T$ is a contraction, then

$$
\|p(T)\| \leq \sup \{|p(z)| \mid z \in \bar{D}\},
$$

for any polynomial $p$, where $D$ is the open unit disc. Thus if $T$ is similar to a contraction, then there exists a constant $M$ such that

$$
\|p(T)\| \leq M \sup \{|p(z)| \mid z \in \bar{D}\},
$$

for all polynomials $p$. In [H2] (problem number six), Halmos asked if the converse is true; that is, if $T$ satisfies (0.1) (such a $T$ is said to be polynomially bounded), is $T$ similar to a contraction?

Halmos' question has recently been answered in the negative by Pisier (see [Pi]). Thus it becomes of interest to ask what stronger condition, analogous to (0.1), is equivalent to similarity to a contraction.

One of the results in this paper (Theorem $4.4(\mathrm{~b}) \rightarrow(\mathrm{d})$ ) provides a sufficient condition very similar to (0.1). It is shown that, if $\bar{D}$ is replaced by a polygon $\mathcal{P} \subseteq \bar{D}$, that is,

$$
\|p(T)\| \leq M \sup \{|p(z)| \mid z \in \overline{\mathcal{P}}\},
$$

then $T$ is similar to a contraction. In fact, (0.2) is shown (Theorem 4.4) to be equivalent to $T$ being similar to a contraction with numerical range contained in a

Received by the editors August 28, 1996.

1991 Mathematics Subject Classification. Primary 47A05; Secondary 47A60, 47D03, 47A45, 47A10, 47A12.

I am indebted to Vũ Quôc Phóng and Christian Le Merdy for invaluable discussions; in particular, to Christian Le Merdy for sending me a preprint of [LM] and pointing out Lemma 1.6, and to Vũ Quôc Phóng for Lemma 3.13. 
closed polygon contained in $\bar{D}$. This equivalence may be restated in the language of von Neumann's inequality above: $(0.2)$ is equivalent to $T$ being similar to an operator $R$ for which there exists a polygon $\mathcal{P}_{0} \subseteq \bar{D}$ such that

$$
\|p(R)\| \leq \sup \left\{|p(z)| \mid z \in \overline{\mathcal{P}_{0}}\right\}
$$

for all polynomials $p$.

It has long been known [R] that, if $\sigma(T)$ is contained in $D$, then $T$ is similar to a contraction (see Remark 3.2). This sufficient condition says that the peripheral spectrum $\sigma(T) \cap \partial D$ is empty. The polygonal condition (0.2) is shown (Theorem 4.4) to be equivalent to $T$ being polynomially bounded, and having finite peripheral spectrum, with a growth condition

$$
\left\|(w-T)^{-1}\right\| \leq \frac{M}{|w-z|},
$$

for $z \in \sigma(T) \cap \partial D, w$ in a half-plane tangent to the unit circle at $z$. The fact that (0.3) implies that $T$ is similar to a contraction, first attributed to Gilles Cassier, appears in a preprint $[\mathrm{Fr}-\mathrm{M}]$ received after this paper was submitted, using a different proof. For $\sigma(T) \cap \partial D$ equal to a single point, the fact that (0.3) implies that $T$ is similar to a contraction first appears in [LM].

The operator $T$ is power bounded if $\sup \left\{\left\|T^{n}\right\| \mid n \in \mathbf{N}\right\}$ is finite. It was shown in [Fo] (see also [H1]) that there exist power-bounded operators that are not similar to a contraction. A natural analogue of power-boundedness is asking that certain orbits

$$
\left\{T^{n} S x \mid n \in \mathbf{N}\right\},
$$

where $S$ is a bounded operator, be square-summable. In Section III it is shown that, with

$$
S \equiv \prod_{k=1}^{m} \sqrt{\left(e^{i \theta_{k}}-T\right)}, \quad \sigma(T) \cap \partial D=\left\{e^{i \theta_{1}}, \ldots, e^{i \theta_{m}}\right\},
$$

such orbits being square-summable implies that $T$ is similar to a contraction (Theorem 3.6). For Theorem 3.6, it is not necessary that $T$ be polynomially bounded; it is sufficient that $T$ be power bounded (Corollary 3.14).

A short proof of a result due to Le Merdy [LM], characterizing those analytic strongly continuous semigroups that are similar to strongly continuous semigroups of contractions, is given in Section II (Corollary 2.4), when the generator is injective.

The paper concludes with a conjectured extension of some of the results (Conjecture 4.12).

Here is the strategy of this paper. The operator $T$ being polynomially bounded is equivalent to saying that $T$ has an $\mathcal{A}$ functional calculus $f \mapsto f(T)$, where $\mathcal{A}$ is the space of functions analytic in the open unit disc, and continuous on the closed unit disc. Paulsen [Pau1] has shown that $T$ is similar to a contraction if and only if

$$
\left[f_{i, j}\right] \mapsto\left[f_{i, j}(T)\right]
$$

is a bounded map from $M_{n}(\mathcal{A})$ into $M_{n}(B(H))$, uniformly in $n$. It is straightforward (see Lemma 1.6) to show that Paulsen's condition is satisfied when

$$
\langle p(T) x, y\rangle=\int_{|w|=1} p(w)\left\langle G_{1}(w) x, G_{2}(w) y\right\rangle d w,
$$


for $x, y$ in dense sets, $p$ a polynomial, for operator-valued functions $G_{j}$ such that, for some constant $K$,

$$
\int_{|w|=1}\left\|G_{j}(w) x\right\|^{2} d|w| \leq K\|x\|^{2}
$$

for all $x \in H, j=1,2$.

Begin with the (limit of the) Riesz-Dunford functional calculus

$$
p(T) x=\int_{|w|=1} p(w)(w-T)^{-1} x d w=\lim _{R \rightarrow 1^{+}} \int_{|w|=R} p(w)(w-T)^{-1} x d w,
$$

which may be valid only for some $x \in H$; then choose an operator-valued function $G$ such that

(1) $G$ is analytic in the open unit disc and measurable on the boundary, and

(2) $\left[(w-T)^{-1}+G(w)\right]=\left[G_{2}(w)\right]^{*} G_{1}(w)$, for $G_{j}$ as in $(0.5)$.

By (1), we have

$$
p(T) x=\int_{|w|=1} p(w)\left[(w-T)^{-1}+G(w)\right] x d w,
$$

so that we obtain the desired representation (0.4).

This technique also works for other regions besides the unit disc; for example, by replacing the unit disc with a sector in the right half-plane, we may deal with analytic semigroups similarly (Section II).

\section{Preliminaries}

All operators are linear, on a Hilbert space $H$, with inner product \langle\rangle$. B(H)$ is the space of all bounded linear operators from $H$ to $H$. The spectrum of a (possibly unbounded) operator $A$ is denoted by $\sigma(A)$, the resolvent set by $\rho(A)$, the image by $\operatorname{Im}(A)$, the domain by $\mathcal{D}(A)$.

I will follow the presentation of Paulsen's results [Pau1] and [Pau2] given in [LM, Section II]. An isomorphism will be $V \in B(H)$ such that $0 \in \rho(V)$. An operator $T \in B(H)$ is similar to $R \in B(H)$ if there exists an isomorphism $V$ such that $T=V R V^{-1}$.

Definition 1.1. Suppose $K$ is a Hilbert space and $E$ is a subspace of $B(K)$. For $n \in \mathbf{N}$, denote by $M_{n}(E)$ the space of $n \times n$ matrices with entries in $E$, with the operator norm

$$
\left\|\left[\phi_{i, j}\right]\right\|_{M_{n}(E)}^{2} \equiv \sup \left\{\sum_{i}\left\|\left(\sum_{j} \phi_{i, j} x_{j}\right)\right\|^{2} \mid x_{j} \in K, \sum_{j=1}^{n}\left\|x_{j}\right\|^{2} \leq 1\right\} .
$$

$M_{n}(\mathbf{C})$ will simply be denoted $M_{n}$.

Definition 1.2. Suppose $H$ and $K$ are Hilbert spaces and $E$ is a subspace of $B(K)$. Then the linear map $\Lambda: E \rightarrow B(H)$ is completely bounded if there exists a constant $c$ so that for all $n \in \mathbf{N}$,

$$
\left\|\left[\Lambda\left(\phi_{i, j}\right)\right]\right\|_{M_{n}(B(H))} \leq c\left\|\left[\phi_{i, j}\right]\right\|_{M_{n}(E)}, \quad \forall\left[\phi_{i, j}\right] \in M_{n}(E) .
$$

Lemma 1.3 ([Pau2, Theorem 8.1]). Suppose $H$ and $K$ are Hilbert spaces, $B$ is a subalgebra of $B(K)$, and $\Lambda: B \rightarrow B(H)$ is a completely bounded algebra homomorphism. Then there exists an isomorphism $V$ such that

$$
\left\|V^{-1}(\Lambda \phi) V\right\|_{B(H)} \leq\|\phi\|_{B(K)}, \quad \forall \phi \in B .
$$


Definition 1.4. Suppose $T \in B(H)$, and $\mathcal{F}$ is a Banach algebra of complex-valued functions defined on a subset of the complex plane, with $f_{0}(z) \equiv 1$ and $f_{1}(z) \equiv z$ in $\mathcal{F}$. Then an $\mathcal{F}$ functional calculus for $T$ is a continuous algebra homomorphism from $\mathcal{F}$ into $B(H)$, which is traditionally written $f \mapsto f(T)$, such that

(1) $f_{0}(T)=I$, and

(2) $f_{1}(T)=T$.

Suppose now that $A$ is a possibly unbounded operator on $H$, and $\mathcal{F}$ is a Banach algebra of complex-valued functions defined on a subset of the complex plane, with $f_{0}(z) \equiv 1$ and $g_{\lambda}(z) \equiv(\lambda-z)^{-1}$ in $\mathcal{F}$, for some complex $\lambda$. Then an $\mathcal{F}$ functional calculus for $A$ is a continuous algebra homomorphism from $\mathcal{F}$ into $B(H), f \mapsto f(A)$, such that

(1) $f_{0}(A)=I$, and

(2) whenever $g_{\lambda} \in \mathcal{F}$, then $\lambda \in \rho(A)$, and $g_{\lambda}(A)=(\lambda-A)^{-1}$.

It is not hard to show that these two definitions are equivalent when $A=T \in$ $B(H)$ and $\mathcal{F}$ contains both $f_{1}$ and $g_{\lambda}$, for some complex $\lambda$.

There will be two Banach algebras of particular interest. Suppose $\Omega$ is a region in the complex plane whose closure is not the entire plane, with piecewise-smooth boundary $\partial \Omega . H^{\infty}(\Omega)$ is the set of all functions in $L^{\infty}(\bar{\Omega})$ that are analytic in $\Omega$, with the supremum norm, and $\mathcal{A}(\Omega)$ is the subalgebra

$$
\mathcal{A}(\Omega) \equiv\left\{f \in H^{\infty}(\Omega) \cap C(\bar{\Omega}) \mid \lim _{|z| \rightarrow \infty, z \in \bar{\Omega}} f(z) \text { exists }\right\} .
$$

Note that, when $\Omega$ is bounded, $\mathcal{A}(\Omega)$ is simply $H^{\infty}(\Omega) \cap C(\bar{\Omega})$.

The same proof as that of [LM, Lemma 2.2] gives us the following, where we consider $\mathcal{A}(\Omega)$ as a subalgebra of $L^{2}(\bar{\Omega})$ (see also [Pau2]).

Lemma 1.5. For any $n \in \mathbf{N}$ and $\left[f_{i, j}\right] \in M_{n}(\mathcal{A}(\Omega))$,

$$
\left\|\left[f_{i, j}\right]\right\|_{M_{n}(\mathcal{A}(\Omega))}=\sup _{w \in \bar{\Omega}}\left\|\left[f_{i, j}(w)\right]\right\|_{M_{n}} .
$$

Lemma 1.6. Suppose $\Omega$ is a region with nonempty piecewise-smooth boundary $\partial \Omega$ in the complex plane, and $f \mapsto f(A)$ is an $\mathcal{A}(\Omega)$ functional calculus for $A$ such that for $j=1,2$, there exist measurable $G_{j}: \partial \Omega \rightarrow B(H)$, a dense subspace $\mathcal{D} \subseteq H$ and a constant $c$ such that

(1)

$$
\langle f(A) x, y\rangle=\int_{\partial \Omega} f(w)\left\langle G_{1}(w) x, G_{2}(w) y\right\rangle d w
$$

for all $f \in \mathcal{A}(\Omega), x, y \in \mathcal{D} ;$ and

$$
\int_{\partial \Omega}\left\|G_{j}(w) x\right\|^{2} d|w| \leq c\|x\|^{2}
$$

for $j=1,2, x \in \mathcal{D}$.

Then there exists an isomorphism $V$ such that

$$
\left\|V^{-1} f(A) V\right\| \leq\|f\|_{\mathcal{A}(\Omega)}, \quad \forall f \in \mathcal{A}(\Omega) .
$$


Proof. Fix $n \in \mathbf{N}, \vec{x}, \vec{y} \in \mathcal{D}^{n},\left[f_{i, j}\right] \in M_{n}(\mathcal{A}(\Omega))$. We argue as follows, using Lemma 1.5 and the Cauchy inequality:

$$
\begin{aligned}
& \left|\left\langle\left[f_{i, j}\right](A) \vec{x}, \vec{y}\right\rangle\right| \equiv\left|\sum_{i, j}\left\langle f_{i, j}(A) x_{j}, y_{i}\right\rangle\right| \\
& =\left|\int_{\partial \Omega} \sum_{i, j} f_{i, j}(w)\left\langle G_{1}(w) x_{j}, G_{2}(w) y_{i}\right\rangle d w\right| \\
& \leq \int_{\partial \Omega}\left\|\left[f_{i, j}(w)\right]\right\|_{M_{n}}\left(\sum_{j}\left\|G_{2}(w) x_{j}\right\|^{2}\right)^{1 / 2}\left(\sum_{i}\left\|G_{1}(w) y_{i}\right\|^{2}\right)^{1 / 2} d w \\
& \leq \sup _{w \in \partial \Omega}\left\|\left[f_{i, j}(w)\right]\right\|_{M_{n}}\left(\int_{\partial \Omega} \sum_{j}\left\|G_{2}(w) x_{j}\right\|^{2} d w\right)^{1 / 2}\left(\int_{\partial \Omega} \sum_{i}\left\|G_{1}(w) y_{i}\right\|^{2} d w\right)^{1 / 2} \\
& \leq c\left\|\left[f_{i, j}\right]\right\|_{M_{n}(\mathcal{A}(\Omega))}\left(\sum_{j}\left\|x_{j}\right\|^{2}\right)^{1 / 2}\left(\sum_{i}\left\|y_{i}\right\|^{2}\right)^{1 / 2} \cdot
\end{aligned}
$$

Since $\mathcal{D}$ is dense, this proves that for any $n \in \mathbf{N},\left[f_{i, j}\right] \in M_{n}(\mathcal{A}(\Omega))$,

$$
\left\|\left[f_{i, j}(A)\right]\right\|_{M_{n}(B(H))} \leq c\left\|\left[f_{i, j}\right]\right\|_{M_{n}(\mathcal{A}(\Omega))},
$$

where $\mathcal{A}(\Omega)$ is considered a subalgebra of $B\left(L^{2}(\bar{\Omega})\right)$.

The conclusion now follows from Lemma 1.3.

Definition 1.7. For $0<\psi \leq \pi, S_{\psi}$ is the open sector $\left\{r e^{i \phi}|| \phi \mid<\psi, r>0\right\}$. A linear operator $A$ is of type $\theta(0 \leq \theta<\pi)$ if, whenever $\theta<\psi<\pi$, there exists a constant $M_{\psi}$ such that $\sigma(A) \subseteq \overline{S_{\psi}}$ and

$$
\left\|(w-A)^{-1}\right\| \leq \frac{M_{\psi}}{|w|}, \forall w \notin \overline{S_{\psi}} .
$$

Lemma 1.8. Suppose $0 \leq \theta<\frac{\pi}{2}, \theta<\psi<\pi, A$ is injective and densely defined and has an $\mathcal{A}\left(S_{\frac{\pi}{2}}\right)$ functional calculus, and $A$ is of type $\theta$. Then $A$ has an $H^{\infty}\left(S_{\psi}\right)$ functional calculus.

Proof. By [LM, Theorem 2.5(ii) $\rightarrow$ (i) and (2.6)], $A$ has bounded imaginary powers. By [M, Section 8], the result follows.

\section{HolomorPhic SEMigroups AND SIMILARITY}

See $[\mathrm{G}],[\mathrm{Paz}]$ or $[\mathrm{VC}]$ for basic material on strongly continuous semigroups and fractional powers.

A strongly continuous semigroup $\{T(t)\}_{t \geq 0}$ is analytic if, for some $\theta>0$, it extends to a family of operators $\{T(z)\}_{z \in S_{\theta}}$ such that, for $0<\phi<\theta, z \mapsto T(z)$ is an analytic map from $S_{\phi}$ into $B(H)$ that is strongly continuous on $\overline{S_{\phi}}$. It is bounded if $\left\{\|T(z)\| \mid z \in S_{\phi}\right\}$ is bounded, whenever $0<\phi<\theta$. A strongly continuous semigroup generated by $A$ will be denoted $\left\{e^{t A}\right\}_{t \geq 0}$.

Throughout this section, $A$ is a densely defined injective operator. Corollary 2.3 says that if $A$ has an $H^{\infty}\left(S_{\psi}\right)$ functional calculus, for some $\psi<\frac{\pi}{2}$, then $-A$ generates a strongly continuous analytic semigroup that is similar to a strongly continuous analytic semigroup of contractions. Corollary 2.4 characterizes those operators of type less than $\frac{\pi}{2}$ that generate strongly continuous semigroups that 
are similar to a strongly continuous semigroup of contractions; it is necessary and sufficient that $A$ have an $H^{\infty}\left(S_{\psi}\right)$ functional calculus for some $\psi$ between 0 and $\frac{\pi}{2}$.

Corollaries 2.3 and 2.4 essentially are in [LM, Theorems 4.3 and 4.5]. [LM, Theorems 4.3 and 4.5] are more general in that they do not require that $A$ be injective. Corollary 2.3 of this paper is more general in that $A$ is not assumed to be of type $\psi$; however, this is well known and not hard to show. One purpose of this section is to offer a short proof of Corollaries 2.3 and 2.4. The key tool, Lemma 2.2 , that provides a desirable representation, as in Lemma 1.6, of any $H^{\infty}\left(S_{\psi}\right)$ functional calculus will also be used in Section IV.

The following is valid on any Banach space, and is probably well known, but I will include its proof for completeness.

Lemma 2.1. If $-A$ generates a bounded strongly continuous analytic semigroup, then for all nonzero real $r$ and all $x \in H$,

$$
A^{\frac{1}{2}}(i r+A)^{-1} x=\int_{0}^{\infty} e^{-i r t} A^{\frac{1}{2}} e^{-t A} x d t .
$$

Proof. We will do this for $r>0$; it will be clear how to modify the proof for $r<0$.

There exists $\theta>0$ so that $\left\{e^{-z A}\right\}_{z \in S_{\theta}}$ is an analytic family of operators. Fix $\phi$ between 0 and $\theta, x \in H$. Then $-e^{-i \phi} A$ generates a bounded strongly continuous semigroup; thus, $-i r \in \rho(A)$, with

$$
\begin{aligned}
(i r+A)^{-1} x & =e^{-i \phi}\left(i e^{-i \phi} r+e^{-i \phi} A\right)^{-1} x \\
& =e^{-i \phi} \int_{0}^{\infty} e^{-i r\left(e^{-i \phi} t\right)} e^{-\left(e^{-i \phi} t\right) A} x d t \\
& =\int_{e^{-i \phi}[0, \infty)} e^{-i r z} e^{-z A} x d z ;
\end{aligned}
$$

since $\left\{e^{-z A}\right\}_{z \in S_{\theta}}$ is analytic, we may apply $A^{\frac{1}{2}}$ :

$$
A^{\frac{1}{2}}(i r+A)^{-1} x=\int_{e^{-i \phi}[0, \infty)} e^{-i r z} A^{\frac{1}{2}} e^{-z A} x d z
$$

Again because $\left\{e^{-z A}\right\}_{z \in S_{\theta}}$ is analytic, $\left\|A^{\frac{1}{2}} e^{-z A}\right\|$ is $O(1 / \sqrt{|z|})$ in $\overline{S_{\phi}}$. A calculusof-residues argument now implies that

$$
\int_{e^{-i \phi}[0, \infty)} e^{-i r z} A^{\frac{1}{2}} e^{-z A} x d z=\int_{0}^{\infty} e^{-i r t} A^{\frac{1}{2}} e^{-t A} x d t
$$

concluding the proof.

Lemma 2.2. Suppose $0<\psi<\frac{\pi}{2}$ and $A$ has an $H^{\infty}\left(S_{\psi}\right)$ functional calculus. Define

$$
G_{1}(w) \equiv A^{\frac{1}{2}}(A-w)^{-1}, \quad G_{2}(w) \equiv \frac{1}{\pi}\left(A^{*}\right)^{\frac{1}{2}}\left(A^{*}-\bar{w}\right)^{-1} \quad(\psi<|\arg (w)| \leq \pi) .
$$

Then for any $\theta$ such that $\frac{\pi}{2} \geq \theta>\psi$, there exist a dense subspace $\mathcal{D} \subseteq H$ and a constant $c_{\theta}$ such that

(1)

$$
\langle f(A) x, y\rangle=\int_{|\arg (w)|=\theta} f(w)\left\langle G_{1}(w) x, G_{2}(w) y\right\rangle d w
$$

for all $f \in \mathcal{A}\left(S_{\theta}\right), x, y \in \mathcal{D}$; and 
$(2)$

$$
\int_{|\arg (w)|=\theta}\left\|G_{j}( \pm w) x\right\|^{2} d|w| \leq c_{\theta}\|x\|^{2},
$$

for $j=1,2, x \in H$.

Proof. Assertion (1) has been shown for $\theta=\frac{\pi}{2}$ in [Bod2, Lemma 2.11], for a dense subspace $\mathcal{D} \subseteq \mathcal{D}(A)$. A calculus-of-residues argument extends (1) to $\frac{\pi}{2} \geq \theta>\psi$.

Assertion (2) will follow from the Plancherel theorem. We will argue with $G_{1}$; it will be clear how to modify the argument for $G_{2}$.

First note that, since $\frac{\pi}{2} \geq \theta>\psi, e^{i\left(\frac{\pi}{2}-\theta\right)} A$ has an $H^{\infty}\left(S_{\left(\psi+\left(\frac{\pi}{2}-\theta\right)\right)}\right)$ functional calculus and $-e^{i\left(\frac{\pi}{2}-\theta\right)} A$ generates a bounded strongly continuous analytic semigroup. Thus by the "quadratic estimates" guaranteed by [M, Section 8(h)], with $\psi(w) \equiv \sqrt{w} e^{-w}$, there exists a constant $M_{\theta}$ so that

$$
\int_{0}^{\infty}\left\|A^{\frac{1}{2}} e^{-t e^{i\left(\frac{\pi}{2}-\theta\right)} A} x\right\|^{2} d t \leq M_{\theta}\|x\|^{2}, \quad \forall x \in H .
$$

Fix $x \in H$. For any real $r$, by Lemma 2.1, we have

$$
\begin{aligned}
G_{1}\left(-r e^{i \theta}\right) x & =e^{i\left(\frac{\pi}{2}-\theta\right)} A^{\frac{1}{2}}\left(i r+e^{i\left(\frac{\pi}{2}-\theta\right)} A\right)^{-1} x \\
& =e^{i\left(\frac{\pi}{2}-\theta\right)} \int_{0}^{\infty} e^{-i r t} A^{\frac{1}{2}} e^{-t e^{i\left(\frac{\pi}{2}-\theta\right)} A} x d t,
\end{aligned}
$$

so that by the Plancherel theorem,

$$
\begin{aligned}
\int_{\arg (w)=\theta}\left\|G_{1}( \pm w) x\right\|^{2} d|w| & \leq \int_{\mathbf{R}}\left\|G_{1}\left(-r e^{i \theta}\right) x\right\|^{2} d r \\
& =\int_{0}^{\infty}\left\|A^{\frac{1}{2}} e^{-t e^{i\left(\frac{\pi}{2}-\theta\right)} A} x\right\|^{2} d t \leq M_{\theta}\|x\|^{2} .
\end{aligned}
$$

An identical argument shows that

$$
\int_{\arg (w)=-\theta}\left\|G_{1}( \pm w) x\right\|^{2} d|w| \leq M_{\theta}\|x\|^{2}, \quad \forall x \in H .
$$

This proves (2).

Corollary 2.3. Suppose $0<\psi<\frac{\pi}{2}$ and $A$ has an $H^{\infty}\left(S_{\psi}\right)$ functional calculus. Then, for $0<\phi<\frac{\pi}{2}-\psi,-A$ generates a strongly continuous analytic semigroup $\left\{e^{-z A}\right\}_{z \in S_{\phi}}$ that is similar to a strongly continuous analytic semigroup of contractions; that is, there exists an isomorphism $V_{\phi}$ such that

$$
\left\|V_{\phi}^{-1} e^{-z A} V_{\phi}\right\| \leq 1 \text { whenever } z \in S_{\phi} .
$$

Proof. Since $A$ has an $H^{\infty}\left(S_{\psi}\right)$ functional calculus, $-A$ generates a strongly continuous analytic semigroup

$$
e^{-z A} \equiv f_{z}(A)\left(z \in S_{\frac{\pi}{2}-\psi}\right),
$$

where $f_{z}(w) \equiv e^{-z w}($ see [Bod1, Theorem 3.1]).

By Lemmas 1.6 and 2.2, there exists an isomorphism $V_{\phi}$ such that

$$
\left\|V_{\phi}^{-1} f(A) V_{\phi}\right\| \leq\|f\|_{\mathcal{A}\left(S_{\frac{\pi}{2}-\phi}\right)}, \quad \forall f \in \mathcal{A}\left(S_{\frac{\pi}{2}-\phi}\right) .
$$

In particular,

$$
\left\|V_{\phi}^{-1} e^{-z A} V_{\phi}\right\|=\left\|V_{\phi}^{-1} f_{z}(A) V_{\phi}\right\| \leq\left\|f_{z}\right\|_{\mathcal{A}\left(S_{\frac{\pi}{2}-\phi}\right)}=1, \quad \forall z \in S_{\phi} .
$$


Corollary 2.4. Suppose $0<\psi \leq \frac{\pi}{2}$ and $-A$ generates a bounded strongly continuous analytic semigroup $\left\{e^{-z A}\right\}_{z \in S_{\psi}}$. Then the following are equivalent.

(a) $\left\{e^{-t A}\right\}_{t \geq 0}$ is similar to a strongly continuous semigroup of contractions.

(b) For $\frac{\pi}{2}-\psi<\theta<\pi$, A has an $H^{\infty}\left(S_{\theta}\right)$ functional calculus.

(c) For $0<\phi<\psi$ there exists an isomorphism $V_{\phi}$ such that

$$
\left\|V_{\phi}^{-1} e^{-z A} V_{\phi}\right\| \leq 1, \quad \forall z \in S_{\phi} .
$$

Proof. (a) $\rightarrow$ (b). By [d, Lemma 2.3], $A$ has an $\mathcal{A}\left(S_{\frac{\pi}{2}}\right)$ functional calculus. Since $\left\{e^{-z A}\right\}_{z \in S_{\psi}}$ is a bounded strongly continuous analytic semigroup, $A$ is of type $\left(\frac{\pi}{2}-\psi\right)$. Lemma 1.8 now implies that $A$ has an $H^{\infty}\left(S_{\theta}\right)$ functional calculus.

(b) $\rightarrow$ (c) follows from Corollary 2.3 .

(c) $\rightarrow$ (a) is clear.

Remark 2.5. Corollary 2.2 and [Bod2] can be used to give an analogue of Stone's theorem valid for arbitrary strongly continuous groups on Hilbert spaces: $i B$ generates a strongly continuous group of exponential type $\omega$ if and only if for all $\alpha>\omega$ $B$ is similar to an operator with spectrum and numerical range contained in the horizontal strip $\{z \in \mathbf{C}|| \operatorname{Im}(z) \mid<\alpha\}$; see $[\mathrm{d}]$.

\section{ORBITS AND SIMILARITY}

Throughout this section, $T \in B(H), D$ is the open unit disc $\{z \in \mathbf{C}|| z \mid<1\}$ and $\sigma(T) \subseteq \bar{D}$.

Theorem 3.6 and Corollary 3.14 give sufficient conditions for $T$ to be similar to a contraction, requiring some (but not all) orbits of $T,(2 \lambda-T)^{-1}$ and $\left[(2 \lambda-T)^{-1}\right]^{*}$, for $\lambda \in \sigma(T) \cap \partial D$, to be square summable.

Throughout this section, it is not required that $T$ be polynomially bounded.

Lemma 3.1. Suppose that, for $j=1,2, G_{j}: \partial D \rightarrow B(H)$ is measurable, and there exists a constant $M$ such that

$$
\int_{|w|=1}\left\|G_{j}(w) x\right\|^{2} d|w| \leq M\|x\|^{2}, \quad \forall x \in H, \quad j=1,2,
$$

and

$$
\langle p(T) x, y\rangle=\int_{|w|=1} p(w)\left\langle G_{1}(w) x, G_{2}(w) y\right\rangle d w
$$

for all polynomials $p, x, y$ in dense subspaces of $H$.

Then $T$ is similar to a contraction.

Proof. Since the polynomials are a dense subalgebra of $\mathcal{A}(D), T$ has an $\mathcal{A}(D)$ functional calculus, with

$$
\langle f(T) x, y\rangle=\int_{|w|=1} f(w)\left\langle G_{1}(w) x, G_{2}(w) y\right\rangle d w
$$

for all $f \in \mathcal{A}(D)$ and all $x, y$ in dense subspaces of $H$.

By Lemma 1.6, there exists an isomorphism $V$ such that

$$
\left\|V^{-1} T V\right\|=\left\|V^{-1} f_{1}(T) V\right\| \leq\left\|f_{1}\right\|_{\mathcal{A}(D)}=1,
$$

where $f_{1}(z) \equiv z$. 
Remark 3.2. Note that, if $\sigma(T) \subseteq D$, then by taking

$$
G_{1}(w) \equiv(w-T)^{-1}, \quad G_{2}(w) \equiv 1 \quad(|w|=1),
$$

we may immediately conclude from Lemma 3.1 that $T$ is similar to a contraction, as in $[R]$.

In the following, let $m$ be Lebesgue measure on the unit circle.

Lemma 3.3. Suppose $m(\sigma(T) \cap \partial D)=0, x \in H$ and

$$
\sum_{n=0}^{\infty}\left\|T^{n} x\right\|^{2}<\infty
$$

Then

(1)

$$
\int_{|w|=1}\left\|(w-T)^{-1} x\right\|^{2} d|w|=\sum_{n=0}^{\infty}\left\|T^{n} x\right\|^{2} ;
$$

and

(2) for any polynomial $p$,

$$
p(T) x=\int_{|w|=1} p(w)(w-T)^{-1} x d w .
$$

If, in addition, $0 \in \rho(T)$ and $m\left(\sigma\left(T^{-1}\right) \cap \partial D\right)=0$, then

$$
\int_{|w|=1}\left\|\left(w-T^{-1}\right)^{-1} x\right\|^{2} d|w|=\sum_{n=1}^{\infty}\left\|T^{n} x\right\|^{2} .
$$

Proof. For $R \geq 1,0 \leq \theta<2 \pi$, define

$$
G_{R}(\theta) \equiv\left(R e^{i \theta}-T\right)^{-1} x .
$$

I claim that $\left\{G_{R}\right\}_{R>1}$ is Cauchy in $L^{2}([0,2 \pi))$ as $R \rightarrow 1^{+}$. This follows by writing, for $|w|>1$,

$$
(w-T)^{-1}=\sum_{n=0}^{\infty} w^{-(n+1)} T^{n},
$$

so that for $R>1$, we may write $G_{R}$ as a Fourier series:

$$
G_{R}(\theta)=\sum_{n=0}^{\infty} e^{-i(n+1) \theta} R^{-(n+1)} T^{n} x(\theta \in[0,2 \pi)) ;
$$

thus, for $R_{1}, R_{2}>1$,

$$
\begin{aligned}
\left\|G_{R_{1}}-G_{R_{2}}\right\|_{L^{2}([0,2 \pi))}^{2} & =\int_{0}^{2 \pi}\left\|\sum_{n=0}^{\infty} e^{-i(n+1) \theta}\left(R_{1}^{-(n+1)}-R_{2}^{-(n+1)}\right) T^{n} x\right\|^{2} d \theta \\
& =\sum_{n=0}^{\infty}\left(R_{1}^{-(n+1)}-R_{2}^{-(n+1)}\right)^{2}\left\|T^{n} x\right\|^{2},
\end{aligned}
$$

so that dominated convergence implies that

$$
\lim _{R_{1}, R_{2} \rightarrow 1^{+}}\left\|G_{R_{1}}-G_{R_{2}}\right\|_{L^{2}([0,2 \pi))}^{2}=0
$$


proving the claim. This implies that the limit, in $L^{2}([0,2 \pi))$, as $R \rightarrow 1^{+}$, of $G_{R}$ exists. Since $m(\sigma(T) \cap \partial D)=0, G_{R} \rightarrow G_{1}$ a.e. as $R \rightarrow 1^{+}$. Thus

$$
\lim _{R \rightarrow 1^{+}}\left\|G_{R}-G_{1}\right\|_{L^{2}([0,2 \pi))}=0 .
$$

Assertion (1) now follows:

$$
\begin{aligned}
\int_{|w|=1} & \left\|(w-T)^{-1} x\right\|^{2} d|w|=\left\|G_{1}\right\|_{L^{2}([0,2 \pi))}^{2} \\
= & \lim _{R \rightarrow 1^{+}}\left\|G_{R}\right\|_{L^{2}([0,2 \pi))}^{2}=\lim _{R \rightarrow 1^{+}} \sum_{n=0}^{\infty}\left(R^{-(n+1)}\right)^{2}\left\|T^{n} x\right\|^{2}=\sum_{n=0}^{\infty}\left\|T^{n} x\right\|^{2} .
\end{aligned}
$$

For (2), let $p$ be a polynomial, $R \geq 1$, and define

$$
G_{R, p}(\theta) \equiv p\left(R e^{i \theta}\right)\left(R e^{i \theta}-T\right)^{-1} x \quad(0 \leq \theta<2 \pi) .
$$

The same argument as with $G_{R}$, with a little extra calculation, shows that

$$
\lim _{R \rightarrow 1^{+}}\left\|G_{R, p}-G_{1, p}\right\|_{L^{2}([0,2 \pi))}=0 .
$$

Thus, using the Riesz-Dunford functional calculus,

$$
\begin{aligned}
p(T) x & =\lim _{R \rightarrow 1^{+}} \int_{|w|=R} p(w)(w-T)^{-1} x d w \\
& =\lim _{R \rightarrow 1^{+}} \int_{0}^{2 \pi} G_{R, p}(\theta) R e^{i \theta} d \theta \\
& =\int_{0}^{2 \pi} G_{1, p}(\theta) e^{i \theta} d \theta \\
& =\int_{|w|=1} p(w)(w-T)^{-1} x d w,
\end{aligned}
$$

giving us (2).

For assertion (3), rewrite

$$
\begin{aligned}
\int_{|w|=1} & \left\|\left(w-T^{-1}\right)^{-1} x\right\|^{2} d|w|=\int_{|w|=1}\left\|\left(T-\frac{1}{w}\right)^{-1} T x\right\|^{2} d|w| \\
& =\int_{0}^{2 \pi}\left\|\left(T-e^{-i \theta}\right)^{-1} T x\right\|^{2} d \theta=\int_{0}^{2 \pi}\left\|\left(T-e^{i \theta}\right)^{-1} T x\right\|^{2} d \theta \\
& =\int_{|w|=1}\left\|(w-T)^{-1} T x\right\|^{2} d|w|=\sum_{n=0}^{\infty}\left\|T^{n+1} x\right\|^{2}=\sum_{n=1}^{\infty}\left\|T^{n} x\right\|^{2},
\end{aligned}
$$

by (1).

Corollary 3.4. Suppose that $\sigma(T) \cap \partial D=\left\{e^{i \theta_{1}}, \ldots, e^{i \theta_{m}}\right\}, S \in B(H)$, and there exists a constant $M$ such that, for all $x \in H$,

$$
\sum_{n=0}^{\infty}\left\|\left[\left(\left(2 e^{i \theta_{k}}-T\right)^{-1}\right)^{n} S\right] x\right\|^{2} \leq M\|x\|^{2},
$$

for $1 \leq k \leq m$.

Then 
(1) there exists a constant $K$ so that for all $x \in H$,

$$
\int_{|w|=1}\|G(w) x\|^{2} d|w| \leq K\|x\|^{2}
$$

(2) for $1 \leq k \leq m$,

$$
\int_{|w|=1}\left\|H_{k}(w) x\right\|^{2} d|w| \leq M\|x\|^{2}
$$

where

$$
H_{k}(w) \equiv\left[\left(2 e^{i \theta_{k}}-w-T\right)^{-1} S\right] \quad\left(1 \leq k \leq m,|w|=1, w \neq e^{i \theta_{k}}\right),
$$

and

$$
G(w) \equiv\left[\left(\prod_{k=1}^{m}\left(2 e^{i \theta_{k}}-w-T\right)^{-1}\right) S\right] \quad\left(|w|=1, w \neq e^{i \theta_{k}}, 1 \leq k \leq m\right) .
$$

Proof. The assertion about $H_{k}$ follows from Lemma 3.3(3), with $T$ replaced by $\left(T-2 e^{i \theta_{k}}\right)^{-1}$.

Now partition the unit circle into $m$ disjoint $\operatorname{arcs} \Gamma_{1}, \Gamma_{2}, \ldots, \Gamma_{m}$, such that, for each $k, e^{i \theta_{k}}$ is in the interior of $\Gamma_{k}$. For $1 \leq k \leq m$, let

$$
C_{k} \equiv\left[\sup _{w \in \Gamma_{k}} \prod_{j \neq k, 1 \leq j \leq m}\left\|\left(2 e^{i \theta_{j}}-w-T\right)^{-1}\right\|^{2}\right] .
$$

Then

$$
\begin{gathered}
\int_{|w|=1}\|G(w) x\|^{2} d|w|=\sum_{k=1}^{m} \int_{\Gamma_{k}}\|G(w) x\|^{2} d|w| \leq \sum_{k=1}^{m} C_{k} \int_{\Gamma_{k}}\left\|H_{k}(w) x\right\|^{2} d|w| \\
\leq \sum_{k=1}^{m} C_{k} \int_{|w|=1}\left\|H_{k}(w) x\right\|^{2} d|w| \leq\left[M \sum_{k=1}^{m} C_{k}\right]\|x\|^{2} .
\end{gathered}
$$

Lemma 3.5. Suppose $m \in \mathbf{N}$ and $w$ and $\left(\alpha_{k}-w\right)$ are in $\rho(T)$, for $k=1,2, \ldots, m$. Then

$$
\begin{gathered}
{\left[\prod_{k=1}^{m}\left(\alpha_{k}-2 T\right)\right](w-T)^{-1} \prod_{k=1}^{m}\left(\alpha_{k}-w-T\right)^{-1}} \\
=(w-T)^{-1}+\left(\alpha_{m}-w-T\right)^{-1}\left[I+\sum_{j=1}^{m-1}\left(\prod_{i=j}^{m-1}\left(\alpha_{i+1}-2 T\right)\left(\alpha_{i}-w-T\right)^{-1}\right)\right] .
\end{gathered}
$$

Proof. This will follow by induction on $m$. For $m=1$, the assertion follows from the resolvent identity

$$
\left(\alpha_{1}-2 T\right)(w-T)^{-1}\left(\alpha_{1}-w-T\right)^{-1}=(w-T)^{-1}+\left(\alpha_{1}-w-T\right)^{-1} .
$$


Now suppose the assertion is valid for $m=n \in \mathbf{N}$. Then

$$
\begin{aligned}
& {\left[\prod_{k=1}^{n+1}\left(\alpha_{k}-2 T\right)\right](w-T)^{-1} \prod_{k=1}^{n+1}\left(\alpha_{k}-w-T\right)^{-1}} \\
& =\left(\alpha_{n+1}-2 T\right)\left(\alpha_{n+1}-w-T\right)^{-1}\left[(w-T)^{-1}+\left(\alpha_{n}-w-T\right)^{-1}\right. \\
& \left.+\left(\alpha_{n}-w-T\right)^{-1} \sum_{j=1}^{n-1}\left(\prod_{i=j}^{n-1}\left(\alpha_{i+1}-2 T\right)\left(\alpha_{i}-w-T\right)^{-1}\right)\right] \\
& =\left(\left(\alpha_{n+1}-w-T\right)+(w-T)\right)\left(\alpha_{n+1}-w-T\right)^{-1}(w-T)^{-1} \\
& +\left(\alpha_{n+1}-2 T\right)\left(\alpha_{n+1}-w-T\right)^{-1}\left[\left(\alpha_{n}-w-T\right)^{-1}\right. \\
& \left.+\left(\alpha_{n}-w-T\right)^{-1} \sum_{j=1}^{n-1}\left(\prod_{i=j}^{n-1}\left(\alpha_{i+1}-2 T\right)\left(\alpha_{i}-w-T\right)^{-1}\right)\right] \\
& =(w-T)^{-1}+\left(\alpha_{n+1}-w-T\right)^{-1} \\
& +\left(\alpha_{n+1}-w-T\right)^{-1}\left[\left(\alpha_{n+1}-2 T\right)\left(\alpha_{n}-w-T\right)^{-1}\right. \\
& \left.+\left(\alpha_{n+1}-2 T\right)\left(\alpha_{n}-w-T\right)^{-1} \sum_{j=1}^{n-1}\left(\prod_{i=j}^{n-1}\left(\alpha_{i+1}-2 T\right)\left(\alpha_{i}-w-T\right)^{-1}\right)\right] \\
& =(w-T)^{-1}+\left(\alpha_{n+1}-w-T\right)^{-1} \\
& +\left(\alpha_{n+1}-w-T\right)^{-1}\left[\left(\alpha_{n+1}-2 T\right)\left(\alpha_{n}-w-T\right)^{-1}\right. \\
& \left.+\sum_{j=1}^{n-1}\left(\prod_{i=j}^{n}\left(\alpha_{i+1}-2 T\right)\left(\alpha_{i}-w-T\right)^{-1}\right)\right] \\
& =(w-T)^{-1}+\left(\alpha_{n+1}-w-T\right)^{-1} \\
& +\left(\alpha_{n+1}-w-T\right)^{-1}\left[\sum_{j=1}^{n}\left(\prod_{i=j}^{n}\left(\alpha_{i+1}-2 T\right)\left(\alpha_{i}-w-T\right)^{-1}\right)\right],
\end{aligned}
$$

completing the induction.

In the following, note that we are not assuming that $T$ is polynomially bounded or power bounded. All we need are square roots of $(z-T)$, for $z \in \partial D \cap \sigma(T)$.

Theorem 3.6. Suppose that $T$ has no eigenvalues on the unit circle, $\sigma(T) \cap \partial D=$ $\left\{e^{i \theta_{1}}, \ldots, e^{i \theta_{m}}\right\}$, and there exist a square root $\sqrt{\left(e^{i \theta_{k}}-T\right)} \in B(H)$ of $\left(e^{i \theta_{k}}-T\right)$ for $1 \leq k \leq m$, and a constant $M$ so that, for every $x \in H$,

$$
\begin{gathered}
\sum_{n=0}^{\infty}\left\|T^{n}\left(\prod_{j=1}^{m} \sqrt{e^{i \theta_{j}}-T}\right) x\right\|^{2} \leq M\|x\|^{2} \\
\sum_{n=0}^{\infty}\left\|\left(\left(2 e^{i \theta_{k}}-T\right)^{-1}\right)^{n}\left(\prod_{j=1}^{m} \sqrt{e^{i \theta_{j}}-T}\right) x\right\|^{2} \leq M\|x\|^{2}
\end{gathered}
$$


and

$$
\sum_{n=0}^{\infty}\left\|\left[\left(\left(2 e^{i \theta_{k}}-T\right)^{-1}\right)^{n}\left(\prod_{j=1}^{m} \sqrt{e^{i \theta_{j}}-T}\right)\right]^{*} x\right\|^{2} \leq M\|x\|^{2}
$$

for $1 \leq k \leq m$.

Then $T$ is similar to a contraction.

Proof. Let

$$
\begin{gathered}
S \equiv\left(\prod_{j=1}^{m} \sqrt{e^{i \theta_{j}}-T}\right), \\
G_{1}(w) \equiv 2^{m}(w-T)^{-1} S(w \in \rho(T)),
\end{gathered}
$$

and

$$
G_{2}(w) \equiv\left[\left(\prod_{k=1}^{m}\left(2 e^{i \theta_{k}}-w-T\right)^{-1}\right) S\right]^{*} \quad\left(\left(2 e^{i \theta_{k}}-w\right) \in \rho(T), 1 \leq k \leq m\right) .
$$

By (3.7) and Lemma 3.3(1), for $j=1$, and (3.9) and Corollary 3.4(1), for $j=2$, there exists a constant $K$ such that

$$
\int_{|w|=1}\left\|G_{j}(w) x\right\|^{2} d|w| \leq K\|x\|^{2}, \quad \forall x \in H, j=1,2 .
$$

By Lemma 3.5, for $w \in \rho(T) \cap \partial D$,

$$
\left[G_{2}(w)\right]^{*} G_{1}(w)=(w-T)^{-1}+G(w),
$$

where

$$
G(w) \equiv\left(2 e^{i \theta_{m}}-w-T\right)^{-1}\left[I+\sum_{j=1}^{m-1}\left(\prod_{i=j}^{m-1}\left(2 e^{i \theta_{i+1}}-2 T\right)\left(2 e^{i \theta_{i}}-w-T\right)^{-1}\right)\right] .
$$

Let $p$ be a polynomial. After a partial-fractions decomposition, (3.8) and Corollary 3.4(2) imply that, for all $x \in H$,

$$
\int_{|w|=1}\|p(w) G(w) S x\|^{2} d|w|<\infty .
$$

Since $w \mapsto p(w) G(w) S x$ is analytic in $|w|<1$, it follows that

$$
\int_{|w|=1} p(w) G(w) S x d w=0
$$

Thus, by (3.11), (3.12) and Lemma 3.3(2), for any $x, z \in H$,

$$
\begin{aligned}
\left\langle p(T) x, S^{*} z\right\rangle & =\langle p(T) S x, z\rangle \\
& =\int_{|w|=1} p(w)\left\langle\left[(w-T)^{-1}+G(w)\right] S x, z\right\rangle d w \\
& =\int_{|w|=1} p(w)\left\langle G_{1}(w) S x, G_{2}(w) z\right\rangle d w \\
& =\int_{|w|=1} p(w)\left\langle G_{1}(w) x, G_{2}(w) S^{*} z\right\rangle d w .
\end{aligned}
$$


Note that, since $S$ is injective, $\operatorname{Im}\left(S^{*}\right)$ is dense. Thus the conclusion of the theorem follows from Lemma 3.1 and (3.10).

Lemma 3.13. If $T$ is power bounded, then for any $\theta \in \mathbf{R}$, there exists a square root $\sqrt{\left(e^{i \theta}-T\right)} \in B(H)$ of $\left(e^{i \theta}-T\right)$.

Proof. By switching to the equivalent (Banach space) norm

$$
\||x|\| \equiv \sup _{n-1 \in \mathbf{N}}\left\|T^{n} x\right\|
$$

we may assume $T$ is a contraction. Then, for $s \geq 0$,

$$
\left\|e^{s(T-1)}\right\|=e^{-s}\left\|e^{s T}\right\| \leq e^{-s} e^{s\|T\|} \leq 1
$$

that is, $(T-1)$ generates a strongly continuous semigroup of contractions. This implies (see, e.g., [Paz, Chapter 2.6]) that $(1-T)$ has a square root given by

$$
\sqrt{(1-T)} \equiv \int_{0}^{\infty}(\lambda+(1-T))^{-1}(1-T) \frac{d \lambda}{\pi \sqrt{\lambda}} .
$$

For arbitrary $\theta \in \mathbf{R}$, define

$$
\sqrt{\left(e^{i \theta}-T\right)} \equiv e^{i \frac{\theta}{2}} \sqrt{\left(1-e^{-i \theta} T\right)}
$$

since $e^{-i \theta} T$ is also power bounded.

Corollary 3.14. Suppose that $T$ is power bounded and has no eigenvalues on the unit circle, $\sigma(T) \cap \partial D=\left\{e^{i \theta_{1}}, \ldots, e^{i \theta_{m}}\right\}$ and there exists a constant $M$ so that, for every $x \in H$,

$$
\begin{gathered}
\sum_{n=0}^{\infty}\left\|T^{n}\left(\prod_{j=1}^{m} \sqrt{e^{i \theta_{j}}-T}\right) x\right\|^{2} \leq M\|x\|^{2} \\
\sum_{n=0}^{\infty}\left\|\left(\left(2 e^{i \theta_{k}}-T\right)^{-1}\right)^{n}\left(\prod_{j=1}^{m} \sqrt{e^{i \theta_{j}}-T}\right) x\right\|^{2} \leq M\|x\|^{2}
\end{gathered}
$$

and

$$
\sum_{n=0}^{\infty}\left\|\left[\left(\left(2 e^{i \theta_{k}}-T\right)^{-1}\right)^{n}\left(\prod_{j=1}^{m} \sqrt{e^{i \theta_{j}}-T}\right)\right]^{*} x\right\|^{2} \leq M\|x\|^{2}
$$

for $1 \leq k \leq m$.

Then $T$ is similar to a contraction.

Proof. Theorem 3.6 and Lemma 3.13. 
Example 3.15. Define $(T f)(z) \equiv z f(z)$, on $H \equiv L^{2}(\bar{D}, \mu)$. For any $f \in H$ and $e^{i \theta_{k}} \in \partial D$, by monotone convergence,

$$
\begin{aligned}
\sum_{n=0}^{\infty}\left\|T^{n}\left(\prod_{k=1}^{m} \sqrt{e^{i \theta_{k}}-T}\right) f\right\|^{2} & =\sum_{n=0}^{\infty} \int_{\bar{D}}|z|^{2 n}\left(\prod_{k=1}^{m}\left|e^{i \theta_{k}}-z\right|\right)|f(z)|^{2} d \mu(z) \\
& =\int_{\bar{D}} \sum_{n=0}^{\infty}|z|^{2 n}\left(\prod_{k=1}^{m}\left|e^{i \theta_{k}}-z\right|\right)|f(z)|^{2} d \mu(z) \\
& =\int_{\bar{D}}\left(1-|z|^{2}\right)^{-1}\left(\prod_{k=1}^{m}\left|e^{i \theta_{k}}-z\right|\right)|f(z)|^{2} d \mu(z) .
\end{aligned}
$$

Similarly, for $1 \leq k \leq m$,

$$
\begin{aligned}
& \sum_{n=0}^{\infty}\left\|\left(\left(2 e^{i \theta_{k}}-T\right)^{-1}\right)^{n}\left(\prod_{j=1}^{m} \sqrt{e^{i \theta_{j}}-T}\right) f\right\|^{2} \\
& =\int_{\bar{D}}\left[\frac{\left|2 e^{i \theta_{k}}-z\right|^{2}}{\left(\left|2 e^{i \theta_{k}}-z\right|^{2}-1\right)}\right]\left(\prod_{j=1}^{m}\left|e^{i \theta_{j}}-z\right|\right)|f(z)|^{2} d \mu(z) .
\end{aligned}
$$

Calculation now shows that (3.7)-(3.9) of Theorem 3.6 will be satisfied if and only if the support of $\mu$ is contained in a polygon $\mathcal{P}$ such that $\mathcal{P} \cap \partial D=\left\{e^{i \theta_{1}}, \ldots, e^{i \theta_{m}}\right\}$.

\section{Finite PERIPHERAL SPECTRUM AND POLYGONAL SIMILARITy}

Throughout this section, as in Section III, $T \in B(H)$ and $\sigma(T) \subseteq \bar{D}$. Assume also that $T$ has no eigenvalues on the unit circle $\partial D$. "Polygon" will mean closed polygon.

Theorem 4.4 gives some conditions, each of which is equivalent to $T$ being similar to a contraction with numerical range contained in a polygon $\mathcal{P} \subseteq \bar{D}$. One equivalent condition is that $\sigma(T) \cap \partial D$ be finite, with an $O\left(\frac{1}{|w-z|}\right)$ growth condition on the resolvent $\left\|(w-T)^{-1}\right\|$, for $z \in \sigma(T) \cap \partial D$ and $w$ in the half-plane tangent to the unit circle at $z$ (Theorem 4.4(a)). This is equivalent to an analogue of polynomial boundedness, with the unit disc replaced by a polygon $\mathcal{P}_{1}$ contained in the closed unit disc (Theorem 4.4(b)), which, in turn, is equivalent to $T$ being similar to an operator $R$ such that

$$
\|p(R)\| \leq \sup \left\{|p(z)| \mid z \in \mathcal{P}_{2}\right\}
$$

for all polynomials $p$, where $\mathcal{P}_{2}$ is a polygon contained in the closed unit disc (Theorem 4.4(c); compare with von Neumann's inequality).

Lemma 4.1. Suppose there exists a constant $M$ such that

$$
\left\|(w-T)^{-1}\right\| \leq \frac{M}{|w-1|},
$$

whenever $\operatorname{Re}(w)>1$, and $T$ is polynomially bounded.

Then, for all $\psi>(\arctan M), T$ has an $H^{\infty}\left(1-S_{\psi}\right)$ functional calculus.

Proof. Let $A \equiv(1-T)$. The spectral condition says that

$$
\left\|(w-A)^{-1}\right\| \leq \frac{M}{|w|}
$$


whenever $\operatorname{Re}(w)<0$. By writing down a power series for the resolvent

$$
(A-(x+i y))^{-1} \equiv \sum_{k=0}^{\infty} x^{k}(A-i y)^{-(k+1)}(|x|<|y| / M, y \in \mathbf{R}),
$$

it is straightforward to verify that $A$ is of type $(\arctan M)$. Since $T$ is polynomially bounded, it has an $\mathcal{A}(D)$ functional calculus. Thus $A$ has an $\mathcal{A}(1+D)$ functional calculus; since $(1+D) \subseteq S_{\frac{\pi}{2}}$, it follows that $A$ has an $\mathcal{A}\left(S_{\frac{\pi}{2}}\right)$ functional calculus. Lemma 1.8 now implies that $A$ has an $H^{\infty}\left(S_{\psi}\right)$ functional calculus, so that $T=1-A$ has an $H^{\infty}\left(1-S_{\psi}\right)$ functional calculus.

Lemma 4.2. Suppose $A$ is injective and of type $\theta<\pi$, and $\theta<\psi<\pi$. Then

$$
\lim _{\epsilon \rightarrow 0^{+}} \int_{\Gamma_{\psi, \epsilon}}\left\|A^{\frac{1}{2}}(w-A)^{-1}\right\| d|w|=0,
$$

where

$$
\Gamma_{\psi, \epsilon} \equiv\left\{\epsilon e^{i \phi} \mid \psi \leq \phi \leq 2 \pi-\psi\right\} .
$$

Proof. The square root $A^{\frac{1}{2}}$ is of type $\frac{\theta}{2}$ (see [K]); thus, for $w \in \Gamma_{\psi, \epsilon}$, denoting by $\sqrt{w}$ the principal square root of $w$, we may write

$$
\begin{aligned}
\left\|A^{\frac{1}{2}}(w-A)^{-1}\right\| & =\left\|\left(A^{\frac{1}{2}}+\sqrt{w}-\sqrt{w}\right)\left(\sqrt{w}+A^{\frac{1}{2}}\right)^{-1}\left(\sqrt{w}-A^{\frac{1}{2}}\right)^{-1}\right\| \\
& \leq\left\|\left(\sqrt{w}-A^{\frac{1}{2}}\right)^{-1}\right\|+\left\|\sqrt{w}\left(\sqrt{w}+A^{\frac{1}{2}}\right)^{-1}\right\|\left\|\left(\sqrt{w}-A^{\frac{1}{2}}\right)^{-1}\right\| \\
& =O\left(|\sqrt{w}|^{-1}\right),
\end{aligned}
$$

for $w \notin \overline{S_{\psi}}$. Thus there exists a constant $M_{\psi}$, independent of $\epsilon$, such that

$$
\int_{\Gamma_{\psi, \epsilon}}\left\|A^{\frac{1}{2}}(w-A)^{-1}\right\| d|w| \leq((2 \pi-2 \psi) \epsilon)\left(\frac{M_{\psi}}{\sqrt{\epsilon}}\right)=2 M_{\psi}(\pi-\psi) \sqrt{\epsilon},
$$

concluding the proof.

Definition 4.3. The numerical range of $R \in B(H)$ is

$$
\text { n.r. }(R) \equiv\{\langle R x, x\rangle \mid x \in H,\|x\|=1\} .
$$

Theorem 4.4. The following are equivalent.

(a) $T$ is polynomially bounded, $\sigma(T) \cap \partial D$ is finite, and there exists a constant $M_{1}$ such that, whenever $z \in \sigma(T) \cap \partial D$, then

$$
\left\|(w-T)^{-1}\right\| \leq \frac{M_{1}}{|w-z|}
$$

for $w$ in the half-plane tangent to the unit circle at $z$.

(b) There exist a polygon $\mathcal{P}_{1} \subseteq \bar{D}$ and a constant $M_{2}$ such that

$$
\|p(T)\| \leq M_{2} \sup \left\{|p(z)| \mid z \in \mathcal{P}_{1}\right\}
$$

for all polynomials $p$.

(c) There exist a polygon $\mathcal{P}_{2} \subseteq \bar{D}$ and an isomorphism $V$ such that

$$
\left\|V^{-1} p(T) V\right\| \leq \sup \left\{|p(z)| \mid z \in \mathcal{P}_{2}\right\},
$$

for all polynomials $p$.

(d) $T$ is similar to a contraction whose numerical range is contained in a polygon $\mathcal{P}_{2} \subseteq \bar{D}$. 
Proof. (a) $\rightarrow$ (c). We will construct a polygon $\mathcal{P}_{2}$ that satisfies Lemma 1.6, with $\Omega$ replaced by the interior of $\mathcal{P}_{2}$. More precisely, we will piece together its boundary, $\Gamma$, using Lemmas 2.2 and 4.1 .

Let $\left\{e^{i \theta_{1}}, \ldots, e^{i \theta_{m}}\right\}$ equal $\sigma(T) \cap \partial D$.

Fix $k$ such that $1 \leq k \leq m$. If $\operatorname{Re}(w)>1$, then $e^{i \theta_{k}} w$ is in the half-plane tangent to $e^{i \theta_{k}}$. Thus

$$
\left\|\left(w-e^{-i \theta_{k}} T\right)^{-1}\right\|=\left\|\left(e^{i \theta_{k}} w-T\right)^{-1}\right\| \leq \frac{M_{1}}{\left|e^{i \theta_{k}} w-e^{i \theta_{k}}\right|}=\frac{M_{1}}{|w-1|} .
$$

It follows from Lemma 4.1 that $A_{k} \equiv\left(1-e^{-i \theta_{k}} T\right)$ has an $H^{\infty}\left(S_{\psi}\right)$ functional calculus, for any $\psi>\left(\arctan M_{1}\right)$.

Let $\theta \equiv \frac{1}{2}\left(\left(\arctan M_{1}\right)+\frac{\pi}{2}\right)$. For $1 \leq k \leq m$, let $\Gamma_{k}$ be the boundary of $e^{i \theta_{k}}\left(1-S_{\theta}\right)$. Since $A_{k}$ is of type $\left(\arctan M_{1}\right), \sigma\left(A_{k}\right) \subseteq S_{\theta} \cup\{0\}$; thus,

$$
\sigma(T) \subseteq e^{i \theta_{k}}\left(1-S_{\theta}\right) \cup\left\{e^{i \theta_{k}}\right\},
$$

for $1 \leq k \leq m$. By putting together pieces of $\Gamma_{k} \cap \bar{D}$, and adding on straight lines contained in $\rho(T) \cap D$, if necessary, we may construct the boundary $\Gamma$ of a polygon $\mathcal{P}_{2}$ that contains $\sigma(T)$, with $\Gamma \cap \sigma(T)=\left\{e^{i \theta_{1}}, \ldots, e^{i \theta_{m}}\right\}$.

Let

$$
S \equiv \prod_{k=1}^{m} \sqrt{\left(e^{i \theta_{k}}-T\right)} .
$$

To use Lemma 1.6, as we used Lemma 3.1 to prove Theorem 3.6, we will need a constant $K$ such that

$$
\int_{\Gamma}\left\|(w-T)^{-1} S x\right\|^{2} d|w| \leq K\|x\|^{2}
$$

and

$$
\int_{\Gamma}\left\|\left[\left(2 e^{i \theta_{k}}-w-T\right)^{-1} S\right]^{*} x\right\|^{2} d|w| \leq K\|x\|^{2},
$$

for all $x \in H, 1 \leq k \leq m$.

By Lemma 2.2, applied to $A_{k}$, there exists a constant $c_{k}$ such that, for any $x \in H$,

$$
\begin{aligned}
\int_{\Gamma_{k}} \| & (w-T)^{-1} \sqrt{\left(e^{i \theta_{k}}-T\right)} x \|^{2} d|w| \\
= & \int_{0}^{\infty}\left\|\left(e^{i \theta_{k}}\left(1-r e^{i \theta}\right)-T\right)^{-1} \sqrt{\left(1-e^{-i \theta_{k}} T\right)} x\right\|^{2} d r \\
& +\int_{0}^{\infty}\left\|\left(e^{i \theta_{k}}\left(1-r e^{-i \theta}\right)-T\right)^{-1} \sqrt{\left(1-e^{-i \theta_{k}} T\right)} x\right\|^{2} d r \\
= & \int_{0}^{\infty}\left\|\left(\left(1-r e^{i \theta}\right)-e^{-i \theta_{k}} T\right)^{-1}\left(A_{k}\right)^{\frac{1}{2}} x\right\|^{2} d r \\
& +\int_{0}^{\infty}\left\|\left(\left(1-r e^{-i \theta}\right)-e^{-i \theta_{k}} T\right)^{-1}\left(A_{k}\right)^{\frac{1}{2}} x\right\|^{2} d r \\
= & \int_{0}^{\infty}\left\|\left(r e^{i \theta}-A_{k}\right)^{-1}\left(A_{k}\right)^{\frac{1}{2}} x\right\|^{2} d r+\int_{0}^{\infty}\left\|\left(r e^{-i \theta}-A_{k}\right)^{-1}\left(A_{k}\right)^{\frac{1}{2}} x\right\|^{2} d r \\
= & \int_{|\arg (w)|=\theta}\left\|\left(w-A_{k}\right)^{-1}\left(A_{k}\right)^{\frac{1}{2}} x\right\|^{2} d|w| \leq c_{k}\|x\|^{2} .
\end{aligned}
$$


For $1 \leq k \leq m, x \in H$, we therefore have

$$
\begin{aligned}
& \int_{\Gamma_{k}}\left\|(w-T)^{-1} S x\right\|^{2} d|w| \\
& \leq\left\|\left[\prod_{j \neq k} \sqrt{\left(e^{i \theta_{k}}-T\right)}\right]\right\| \int_{\Gamma_{k}}\left\|(w-T)^{-1} \sqrt{\left(e^{i \theta_{k}}-T\right)} x\right\|^{2} d|w| \\
& \leq c_{k}\left\|\left[\prod_{j \neq k} \sqrt{\left(e^{i \theta_{k}}-T\right)}\right]\right\|\|x\|^{2} .
\end{aligned}
$$

Since $\overline{\left(\Gamma-\bigcup_{k=1}^{m} \Gamma_{k}\right)} \subseteq \rho(T),(4.5)$ follows.

Since, for any $\epsilon>0, w \mapsto\left[\left(2 e^{i \theta_{k}}-w-T\right)^{-1}\right]^{*}$ is analytic in a neighborhood of $\bar{D} \cap\left\{w|| w-e^{i \theta_{k}} \mid>\epsilon\right\}$, to show (4.6) it is sufficient to show that

$$
\int_{\Gamma_{k}}\left\|\left[\left(2 e^{i \theta_{k}}-w-T\right)^{-1} \sqrt{\left(e^{i \theta_{k}}-T\right)}\right]^{*} x\right\|^{2} d|w| \leq c_{k}\|x\|^{2} .
$$

The calculation follows; note that $A_{k}^{*}$ has the same functional calculi as $A_{k}$, for $1 \leq k \leq m$ :

$$
\begin{aligned}
\int_{\Gamma_{k}} \| & \left.\left(2 e^{i \theta_{k}}-w-T\right)^{-1} \sqrt{\left(e^{i \theta_{k}}-T\right)}\right]^{*} x \|^{2} d|w| \\
= & \int_{0}^{\infty}\left\|\left[\left(2 e^{i \theta_{k}}-e^{i \theta_{k}}\left(1-r e^{i \theta}\right)-T\right)^{-1}\left(A_{k}\right)^{\frac{1}{2}}\right]^{*} x\right\|^{2} d r \\
& +\int_{0}^{\infty}\left\|\left[\left(2 e^{i \theta_{k}}-e^{i \theta_{k}}\left(1-r e^{-i \theta}\right)-T\right)^{-1}\left(A_{k}\right)^{\frac{1}{2}}\right]^{*} x\right\|^{2} d r \\
= & \int_{0}^{\infty}\left\|\left[\left(e^{i \theta_{k}}\left(1+r e^{i \theta}\right)-T\right)^{-1}\left(A_{k}\right)^{\frac{1}{2}}\right]^{*} x\right\|^{2} d r \\
& +\int_{0}^{\infty}\left\|\left[\left(e^{i \theta_{k}}\left(1+r e^{-i \theta}\right)-T\right)^{-1}\left(A_{k}\right)^{\frac{1}{2}}\right]^{*} x\right\|^{2} d r \\
= & \int_{0}^{\infty}\left\|\left[\left(1+r e^{i \theta}-e^{-i \theta_{k}} T\right)^{-1}\left(A_{k}\right)^{\frac{1}{2}}\right]^{*} x\right\|^{2} d r \\
& +\int_{0}^{\infty}\left\|\left[\left(1+r e^{-i \theta}-e^{-i \theta_{k}} T\right)^{-1}\left(A_{k}\right)^{\frac{1}{2}}\right]^{*} x\right\|^{2} d r \\
= & \int_{0}^{\infty}\left\|\left[\left(A_{k}+r e^{i \theta}\right)^{-1}\left(A_{k}\right)^{\frac{1}{2}}\right]^{*} x\right\|^{2} d r+\int_{0}^{\infty}\left\|\left[\left(A_{k}+r e^{-i \theta}\right)^{-1}\left(A_{k}\right)^{\frac{1}{2}}\right]^{*} x\right\|^{2} d r \\
= & \int_{|\arg (w)|=\theta}\left\|\left(A_{k}^{*}+w\right)^{-1}\left(A_{k}^{*}\right)^{\frac{1}{2}} x\right\|^{2} d|w| \leq c_{k}\|x\|^{2},
\end{aligned}
$$

for all $x \in H, 1 \leq k \leq m$, again by Lemma 2.2. Assertion (4.6) follows.

Define $G_{1}, G_{2}$ exactly as in the proof of Theorem 3.6. Assertions (4.5) and (4.6) imply that there exists a constant $M$ so that

$$
\int_{\Gamma}\left\|G_{j}(w) x\right\|^{2} d|w| \leq M\|x\|^{2}
$$

for all $x \in H, j=1,2$. That is, $G_{1}, G_{2}$ satisfy Lemma $1.6(2)$, with $\Omega$ chosen to be the interior of the polygon $\mathcal{P}_{2}$. 
Lemma 1.6(1) must also be verified. Since the polynomials are dense in $\mathcal{A}\left(\mathcal{P}_{2}\right)$, it is sufficient to show that

$$
\langle p(T) x, y\rangle=\int_{\Gamma} p(w)\left\langle G_{1}(w) x, G_{2}(w) y\right\rangle d w,
$$

for all polynomials $p$, all $x \in H$, and all $y \in \operatorname{Im}\left(S^{*}\right)$.

As with the proof of Theorem 3.6, we start with a Riesz-Dunford functional calculus; this time, place $\epsilon$-balls around $e^{i \theta_{k}}, 1 \leq k \leq m$, to form, for $\epsilon>0$, the contour

$$
\Gamma(\epsilon) \equiv \partial\left[\mathcal{P}_{2} \bigcup_{k=1}^{m}\left\{w \in \mathbf{C}|| w-e^{i \theta_{k}} \mid<\epsilon\right\}\right] .
$$

For any $\epsilon>0$, since $\Gamma(\epsilon)$ surrounds $\sigma(T)$, we have

$$
p(T)=\int_{\Gamma(\epsilon)} p(w)(w-T)^{-1} d w
$$

By Lemma 4.2 ,

$$
\int_{\Gamma} p(w)(w-T)^{-1} S x d w=\lim _{\epsilon \rightarrow 0^{+}} \int_{\Gamma(\epsilon)} p(w)(w-T)^{-1} S x d w=p(T) S x,
$$

for any $x \in H$ and any polynomial $p$.

The remainder of the proof is exactly like that of Theorem 3.6, with the polygon $\mathcal{P}_{2}$ replacing the unit disc $D$ and Lemma 1.6 replacing Lemma 3.1, so that (c) follows.

$(\mathrm{c}) \rightarrow(\mathrm{b})$ is clear.

(b) $\rightarrow$ (a). Assertion (b) implies that $T$ has an $\mathcal{A}\left(\mathcal{P}_{1}\right)$ functional calculus $f \mapsto$ $f(T)$, with

$$
\|f(T)\| \leq M_{2} \sup \left\{|f(z)| \mid z \in \mathcal{P}_{1}\right\} \quad \forall f \in \mathcal{A}\left(\mathcal{P}_{1}\right) .
$$

Using the fact that $(w-T)^{-1}=g_{w}(T), g_{w}(z) \equiv(w-z)^{-1}$, I can now assert that

$$
\left\|(w-T)^{-1}\right\| \leq M_{2}\left\|g_{w}\right\|_{\mathcal{A}\left(\mathcal{P}_{1}\right)}=\frac{M_{2}}{d\left(w, \mathcal{P}_{1}\right)},
$$

for any $w \notin \mathcal{P}_{1}$, which implies (a), since $\mathcal{P}_{1}$ is a polygon.

(d) $\rightarrow$ (a). Suppose $R=V^{-1} T V$ is a contraction, with n.r. $(R) \subseteq \mathcal{P}_{2}$, for $\mathcal{P}_{2}$ a polygon. By von Neumann's inequality, $R$, hence $T$, is polynomially bounded. Also, $\sigma(R) \subseteq \mathcal{P}_{2}$, with

$$
\left\|(w-R)^{-1}\right\| \leq \frac{1}{d\left(w, \mathcal{P}_{2}\right)} \forall w \notin \mathcal{P}_{2}
$$

([Paz, Theorem 1.3.9]), which, as in (b) $\rightarrow$ (a), gives us (a).

(c) $\rightarrow$ (d). Let $R \equiv V^{-1} T V$. Clearly $R$ is a contraction.

We will show that $n . r .(R) \subseteq \mathcal{P}_{2}$. Write $\mathcal{P}_{2}$ as an intersection of sectors

$$
\mathcal{P}_{2}=\bigcap_{k=1}^{m} e^{i \theta_{k}}\left(1-\overline{S_{\psi_{k}}}\right)
$$

where $e^{i \theta_{1}}, \ldots, e^{i \theta_{m}}$ are the vertices of $\mathcal{P}_{2}$, and $0<\psi_{k}<\frac{\pi}{2}$, for $1 \leq k \leq m$.

For $1 \leq k \leq m$, define, as in (a) $\rightarrow$ (c),

$$
A_{k} \equiv\left(1-e^{-i \theta_{k}} R\right)
$$


Assertion (c) implies that $-A_{k}$ generates a strongly continuous analytic semigroup $\left\{e^{-z A_{k}}\right\}_{z \in S_{\left(\frac{\pi}{2}-\psi_{k}\right)}}$ of contractions. This implies that

$$
\text { n.r. }\left(A_{k}\right) \subseteq \overline{S_{\psi_{k}}}
$$

for $1 \leq k \leq m$, so that

$$
n . r .(R) \subseteq \bigcap_{k=1}^{m} e^{i \theta_{k}}\left(1-\overline{S_{\psi_{k}}}\right)=\mathcal{P}_{2}
$$

as desired.

Remark 4.7. For $\sigma(T) \cap \partial D$ a single point, the fact that Theorem 4.4(a) implies $T$ is similar to a contraction appears in [LM, Corollary 4.7], except that the growth condition in Theorem 4.4(a) is required for all $w \notin \bar{D}$. However, in [LM, Corollary 4.7], the point on $\sigma(T) \cap \partial D$ could be an eigenvalue of $T$.

Remark 4.8. It should be mentioned that (a)-(d) of Theorem 4.4 are equivalent to

(e) $T$ is polynomially bounded and similar to an operator with numerical range contained in a polygon $\mathcal{P} \subseteq \bar{D}$.

It is clear, by von Neumann's inequality, that $(\mathrm{d}) \rightarrow(\mathrm{e})$, and the proof of $(\mathrm{d}) \rightarrow$ (a) only required (e).

In general, if $T$ is a contraction, then $\operatorname{n.r.}(T) \subseteq \bar{D}$, but the converse is not true; consider

$$
T_{\alpha} \equiv\left[\begin{array}{ll}
0 & \alpha \\
0 & 0
\end{array}\right]
$$

on $\mathbf{C}^{2}$. If $1<\alpha \leq \sqrt{2}$, then $n . r .(T) \subseteq \bar{D}$, but $T$ is not a contraction. Thus the operator that $T$ is similar to, in Theorem $4.4(\mathrm{~d})$, is very well-behaved.

Numerical range and contractions are related in at least two other ways. First, a (possibly unbounded) densely defined operator $A$ has spectrum and numerical range contained in the closed right half-plane $\overline{S_{\frac{\pi}{2}}}$ if and only if $-A$ generates a strongly continuous semigroup of contractions (this is the Lumer-Phillips theorem; see $[\mathrm{G}],[\mathrm{Paz}]$ or $[\mathrm{VC}])$. Second, if $C$ is the Cayley transform $C(z) \equiv(1-z)(z+1)^{-1}$, mapping $S_{\frac{\pi}{2}}$ onto $D$, then $A$ has numerical range contained in $\overline{S_{\frac{\pi}{2}}}$ if and only if $C(A)$ is a (not necessarily everywhere-defined) contraction.

Thus Halmos's original similarity problem may be equivalently stated in terms of numerical range: If $A$ has an $\mathcal{A}\left(\overline{S_{\frac{\pi}{2}}}\right)$ functional calculus, is $A$ similar to an operator with numerical range contained in $\overline{S_{\frac{\pi}{2}}}$ ? (Such an operator is called accretive.) Since Pisier [Pi] has now answered this question in the negative, the next natural question, in the language of numerical range, is the following: If $A$ has an $\mathcal{A}\left(\overline{S_{\frac{\pi}{2}}}\right)$ functional calculus, what additional hypotheses are necessary to make $A$ similar to an operator with numerical range contained in $\overline{S_{\frac{\pi}{2}}}$ ? Le Merdy [LM] showed that it is sufficient that $A$ have an $\mathcal{A}\left(\overline{S_{\theta}}\right)$ functional calculus, for some positive $\theta$ less than $\frac{\pi}{2}$ (see Corollary 2.3).

Remark 4.9. There are curious similarities between Theorems 3.6 and 4.4. In both cases, the proofs invoke Lemma 1.6 with

$$
G_{1}(w) \equiv 2^{m}(w-T)^{-1} S \quad \text { and } \quad G_{2}(w) \equiv\left[\left(\prod_{k=1}^{m}\left(2 e^{i \theta_{k}}-w-T\right)^{-1}\right) S\right]^{*}
$$


where

$$
S \equiv\left(\prod_{j=1}^{m} \sqrt{e^{i \theta_{j}}-T}\right) .
$$

The difference is that, in Theorem 3.6, $\Omega$, from Lemma 1.6, is chosen to be the open unit disc $D$, while in Theorem $4.4, \Omega$ is the interior of a polygon.

Hypotheses (3.7) and (3.9), from Theorem 3.6, are equivalent to

$$
\langle p(T) x, y\rangle=\int_{\partial D} p(w)\left\langle G_{1}(w) x, G_{2}(w) y\right\rangle d w,
$$

for all polynomials $p$ and any $x \in H, y \in \operatorname{Im}\left(S^{*}\right)$, and

$$
\int_{\partial D}\left\|G_{j}(w) x\right\|^{2} d|w| \leq M\|x\|^{2}
$$

for all $x \in H$, while (a)-(c) of Theorem 4.4 are equivalent to

$$
\langle p(T) x, y\rangle=\int_{\partial P} p(w)\left\langle G_{1}(w) x, G_{2}(w) y\right\rangle d w,
$$

for all polynomials $p$ and all $x \in H, y \in \operatorname{Im}\left(S^{*}\right)$, and

$$
\int_{\partial P}\left\|G_{j}(w) x\right\|^{2} d|w| \leq M\|x\|^{2}
$$

for all $x \in H$, for some polygon $\mathcal{P} \subseteq \bar{D}$.

It is therefore natural to conjecture that (3.7) and (3.9), from Theorem 3.6, are equivalent to (a)-(d) of Theorem 4.4. At least, since the conclusion drawn from Lemma 1.6 from (4.11) is stronger than the conclusion drawn from Lemma 1.6 from (4.10), it is natural to conjecture that (a)-(d) of Theorem 4.4 imply (3.7) and (3.9) of Theorem 3.6. But since Lemma 1.6 is not an equivalence, this does not follow.

If $T$ is the normal operator in Example 3.15, then it may be shown that $T$ satisfies (3.8)-(3.10) of Theorem 3.6 if and only if $T$ satisfies (a)-(c) of Theorem 4.4 if and only if the support of $\mu$ is contained in a polygon $\mathcal{P} \subseteq \bar{D}$.

Conjecture 4.12. It is possible that infinite analogues of Theorems 3.6 and 4.4 could be shown by analogous methods.

The hypothesis in Theorem 4.4(a) could be replaced by

$$
\left\|(w-T)^{-1}\right\| \leq \frac{M_{z}}{|w-z|},
$$

for all $z \in \sigma(T) \cap \partial D$, and $w$ in the half-plane tangent to $w$. The set of constants $\left\{M_{z} \mid z \in \sigma(T) \cap \partial D\right\}$ in (4.13) would be unbounded, in general. (4.13) would imply that $\sigma(T) \cap \partial D$ is countable. The polygon $\mathcal{P}_{2}$ of Theorem 4.4(c) would be replaced by a figure whose boundary is an infinite union of chords. Showing that the integral of $\left\|G_{j}(w) x\right\|^{2}$ over its boundary is finite is made much more difficult by the fact that it's an infinite union.

In Theorem 3.6, one could replace the finite peripheral spectrum $\sigma(T) \cap \partial D$ by a peripheral spectrum of (one-dimensional) measure zero, then look for $f \in \mathcal{A}(D)$ and $H(w, T), G(w, T)$ such that

(1) $w \mapsto G(w, T)$ is analytic in $D$;

(2) $\{z \in \partial D \mid f(z)=0\}=\sigma(T) \cap \partial D$;

(3) $w \mapsto H(w, T)$ is analytic in $\{z \in \mathbf{C}|| z \mid>1\} \cup\{z \in \partial D \mid f(z) \neq 0\}$; 
(4) $(f(T))^{2}(w-T)^{-1} H(w, T)=(w-T)^{-1}+G(w, T)$; and

(5) if $G_{1}(w) \equiv(w-T)^{-1} f(T)$ and $G_{2}(w) \equiv[H(w, T) f(T)]^{*}$, then there exists a constant $M$ so that

$$
\int_{|w|=1}\left\|G_{j}(w) x\right\|^{2} d|w| \leq M\|x\|^{2}
$$

for $x \in H, j=1,2$.

In $(3.7)-(3.9), f(z) \equiv \prod_{j=1}^{m} \sqrt{2\left(e^{i \theta_{j}}-z\right)}, H(w, T) \equiv \prod_{k=1}^{m}\left(2 e^{i \theta_{k}}-w-T\right)^{-1}$.

Possible sufficient conditions for $T$ to be similar to a contraction, analogous to (3.7)-(3.9), would be

$$
\sum_{n=0}^{\infty}\left\|T^{n} f(T) x\right\|^{2} \leq M\|x\|^{2},
$$

for all $x \in H$,

$$
\begin{gathered}
\sum_{n=0}^{\infty}\left\|\left(\left(2 e^{i \theta}-T\right)^{-1}\right)^{n} f(T) x\right\|^{2} \leq M\|x\|^{2}, \\
\sum_{n=0}^{\infty}\left\|\left[\left(\left(2 e^{i \theta}-T\right)^{-1}\right)^{n} f(T)\right]^{*} x\right\|^{2} \leq M\|x\|^{2},
\end{gathered}
$$

whenever $x \in H$ and $e^{i \theta} \in \sigma(T) \cap \partial D$.

\section{REFERENCES}

[Be] B. Beauzamy, "Introduction to Operator Theory and Invariant Subspaces," NorthHolland, Amsterdam, 1988. MR 90d:47001

[Bod1] K. Boyadzhiev and R. deLaubenfels, Semigroups and resolvents of bounded variation, imaginary powers and $H^{\infty}$ functional calculi, Semigroup Forum 45 (1992), 372-384. MR 93i: 47018

[Bod2] K. Boyadzhiev and R. deLaubenfels, Spectral theorem for unbounded strongly continuous groups on a Hilbert space, Proc. Amer. Math. Soc. 120 (1994), 127-136. MR 94c:47058

[d] R. deLaubenfels, Strongly continuous groups, similarity and numerical range on a Hilbert space, Taiwanese J. Math. 1 (1997), 127-133. CMP 97:13

[Fo] S. R. Foguel, A counterexample to a problem of Sz.-Nagy, Proc. Amer. Math. Soc. 15 (1964), 788-790. MR 29:2646

[Fr-M] E. Franks and A. McIntosh, Discrete quadratic estimates and holomorphic functional calculi of operators in Banach spaces, Ulmer Seminare über Funktionalanalysis und Differentialgleichungen 1996, 155-172.

[G] J. A. Goldstein, "Semigroups of Linear Operators and Applications," Oxford Univ. Press, New York, 1985. MR 87c:47056

[H1] P. R. Halmos, On Foguel's answer to Nagy's question, Proc. Amer. Math. Soc. 15 (1964), 791-793. MR 29:2647

[H2] P. R. Halmos, Ten problems in Hilbert space, Bull. Amer. Math. Soc. 76 (1970), 887-933. MR 42:5066

[K] T. Kato, Note on fractional powers of linear operators, Proc. Japan Acad. 36 (1960), 94-96. MR 22:12400

[LM] C. Le Merdy, The similarity problem for bounded analytic semigroups on Hilbert space, Semigroup Forum, to appear.

[M] A. McIntosh, Operators which have an $H^{\infty}$ functional calculus, Miniconference on Operator Theory and Partial Differential Equations, Proc. Center Math. Anal., ANU, vol. 14, Australian Nat. Univ., Canberra, 1986, pp. 210-231. MR 88k:47019

[Pau1] V. I. Paulsen, Every completely polynomially bounded operator is similar to a contraction, J. Funct. Anal. 55 (1984), 1-17. MR 86c:47021 
[Pau2] V. I. Paulsen, "Completely Bounded Maps and Dilations," Pitman Research Notes in Math. 146, Longman Sci. Tech., Harlow, and Wiley, New York, 1986. MR 88h:46111

[Paz] A. Pazy, "Semigroups of Linear Operators and Applications to Partial Differential Equations," Springer, New York, 1983. MR 85g:47061

[Pi] G. Pisier, A polynomially bounded operator on Hilbert space which is not similar to a contraction, J. Amer. Math. Soc. 10 (1997), 351-369. MR 97f:47002

[R] G. C. Rota, On models for linear operators, Comm. Pure Appl. Math. 13 (1960), 469-472. MR 22:2898

[VC] J. A. van Casteren, "Generators of Strongly Continuous Semigroups," Research Notes in Mathematics 115, Pitman, Boston, 1985. Zbl.576:47023

Scientia Research Institute, P. O. Box 988, Athens, Ohio 45701

E-mail address: 72260.2403@compuserve.com 